\title{
DESIGN STUDY ON STANDING-WAVE LINEAR ACCELERATOR
}

\author{
N. Nepal, Y. K. Kim, Y. S. Bae, I. S. Ko, M. H. Cho, and W. Namkung \\ Pohang University of Science and Technology, Pohang 790-784, Republic of Korea
}

\begin{abstract}
A compact standing-wave linac has been designed using S-band microwaves for medical and industrial applications. It uses a bi-periodic structure with constant impedance. It consists of a buncher section with three-cells and on-axis coupled cavities with nine-cells. The total length is 740.0 mm including the electron gun. Using SUPERFISH, HFSS and PARMELA codes, it is capable to deliver $4.5 \mathrm{MeV}$ electron beams with pulsed microwaves of $4.8 \mathrm{MW}$ at 2,856 MHz. In this paper, we present the detailed design studies on a compact S-band standing-wave linac.
\end{abstract}

\section{INTRODUCTION}

Although the first RF linear accelerator was installed at Hammersmith Hospital in UK in 1952 [1], the design and manufacture of extremely small, low energy, high current, and high quality linear accelerators are becoming state-ofart due to extensive use for medical and industrial purposes. At present, there are thousands of standing-wave (SW) machines for radiotherapy, radiography, and industrial purposes. Design of such accelerators includes the precise study of dispersion relations of periodic slow-wave structure, resonant cavity, E-GUN, and coupler. They operate on different structure modes. Among them the $\pi$ mode is the one that has often been proposed for standingwave accelerators giving higher shunt impedance. But it increases sensitivity to frequency deviation, dimensional tolerance and cell-to-cell phase deviation. Most of the high-energy accelerators around the world operate on $2 \pi / 3$-mode. It is less sensitive to construction and operational differences compared to that of $\pi$-mode but not compared to $\pi / 2$-mode [2]. $\pi / 2$-mode is the least sensitive and gives the lowest shunt impedance among $\pi$ and $2 \pi / 3$ mode.

Designers prefer the $\pi$-mode structure with double periodicities. The phase shift per cavity in those designs is really $\pi / 2$, and the shunt impedance is slightly lower than pure $\pi$-mode. Such bi-periodic structures are side-coupled and on-axis coupled structures. In side-coupled structure, half of the cavities are not on-axis with electron beams. This structure increases effective diameter and number of matching and assembly steps [3]. These reasons led present design to use the bi-periodic on-axis coupled structure. It gives the shunt impedance similar to that of $\pi$-mode and the phase shift per cavity of $\pi / 2$. Present work presents detailed design and simulation results on a compact S-band standing wave electron linear accelerator, which can be used for food irradiation, recycling, and medical applications.

\section{DESIGN CONCEPT}

The available S-band (2856 MHz) microwave source and double periodic structure are chosen as first basic parameter choices. Thus for a fixed phase shift $\phi, \mathrm{RF}$ frequency $\omega$ and particle velocity $v_{\mathrm{p}} \sim \mathrm{c}$ the length of one cavity, $l$ is given by $\phi \mathrm{c} / \omega$. But, for synchronism condition it is given by $(\phi+2 \pi \mathrm{n}) \beta \lambda / 2 \pi$. Where $\beta$ is the particle relative velocity, $\lambda$ is the RF wavelength, and $n$ is an integer. The estimation of the accelerating field is very important for design. In a short standing wave linac, it is given by $\left(\mathrm{p}_{0} \mathrm{R}_{\mathrm{s}} / \mathrm{L}\right)^{1 / 2}$, for peak input $\mathrm{RF}$ power $\mathrm{P}_{0}$, accelerator length $\mathrm{L}$, and shunt impedance $\mathrm{R}_{\mathrm{s}}$. However, the power loss should be taken into account. The cutoff frequency depends upon the wave-guide diameter, while the bandwidth depends on the cavity to bore radius ratio. The bandwidth can be calculated from the dispersion relation. The dispersion relation is approximated by [4]

$$
\omega=\omega_{\text {cell }} \sqrt{1-\alpha \cos \mathrm{k} /},
$$

where $\alpha$ is the coupling coefficient. From Eq. (1), the group velocity is given by

$$
v_{\mathrm{g}}=\frac{1}{2} \alpha \omega / \sin \mathrm{k} / \text {. }
$$

In the case of electrical coupling ( $\alpha$ is positive), the phase and energy propagate in the same direction. In the case of magnetic coupling ( $\alpha$ is negative), the phase and energy propagate in the opposite direction. The group velocity has a maximum value at $\pi / 2$-mode, and the attenuation becomes minimum. Thus knowing the group velocity and structure attenuation, the bore radius of the cavity can be fixed. The choice of the disk thickness involves a compromise between the use of the thin disk to increase the shunt impedance and thick disk to reduce danger of electric breakdowns, to improve heat transfer characteristics, and to increase mechanical strength. The disk thickness should be more than $3.0 \mathrm{~mm}$. Operation at a particular mode without excitation of its neighboring mode requires that the quality factor, $\mathrm{Q}$ of the structure be sufficiently high to prevent overlapping of the resonant modes [5]. From the dispersion relation we can get

$$
\begin{aligned}
& Q_{0, \pi}>\frac{4(N-1)^{2}}{\alpha \pi^{2}}, \\
& Q_{\pi / 2}>\frac{4(N-1)}{\alpha \pi},
\end{aligned}
$$

where $\mathrm{N}$ is the number of cavities in the structure. Thus $\pi / 2$ phase shift is appropriate for design. 
The optimization of the beam loading is also important for design. For medical application the maximization of the formula giving the dose rate output becomes [6]

$$
\mathrm{I}_{\mathrm{opt}}=\frac{2}{\mathrm{~m}} \sqrt{\frac{\beta_{\mathrm{opt}} \mathrm{p}_{0}}{\mathrm{R}_{\mathrm{s}} \mathrm{L}}},
$$

where $\beta_{\text {opt }}=(\mathrm{m}+1) /(\mathrm{m}-1), \mathrm{m}=3$ for $4 \mathrm{MeV}$ accelerator.

\section{$\underline{\text { Table I- Accelerator Parameters }}$}

$\begin{array}{ll}\text { Beam energy } & 4.5 \mathrm{MeV} \\ \text { RF frequency } & 2,856 \mathrm{MHz} \\ \text { Input RF power } & 4.8 \mathrm{MW} \\ \text { Beam current (peak) } & 300 \mathrm{~mA} \\ \text { Beam power (peak) } & 1.4 \mathrm{MW} \\ \text { Pulse width } & 5 \mu \mathrm{sec} \\ \text { Repetition Rate } & 960 \mathrm{pps} \\ \text { Beam power (average) } & 6.5 \mathrm{~kW} \\ \text { Beam diameter } & 4.6 \mathrm{~mm} \\ \text { Injection energy } & 25 \mathrm{keV} \\ \text { Structure type } & \mathrm{Bi}-\mathrm{periodic} \\ \text { Number of cell } & 3+9 \\ \text { Average field } & 34 \mathrm{MV} / \mathrm{m} \\ \text { Shunt impedance } & 85 \mathrm{M} \Omega / \mathrm{m} \\ \text { Quality factor } & 17,379 \\ \text { Heat-loss } & 16.5 \mathrm{~kW}\end{array}$

\section{E-GUN}

The electron source is a Pierce-type diode with a $5.0 \mathrm{~mm}$ radius planer cathode. It provides $1.02 \mathrm{~A}$ current at $25 \mathrm{kV}$. By the E-GUN code perveance is of $0.26 \times 10^{-6}\left[\mathrm{~A} / \mathrm{V}^{3 / 2}\right]$ with $\epsilon_{\mathrm{n}}=4.76 \pi \mathrm{mm}$-mrad. Simulation result of electron beam trajectories in the GUN is shown in Fig. 1, where one unit is equal to $0.5 \mathrm{~mm}$.

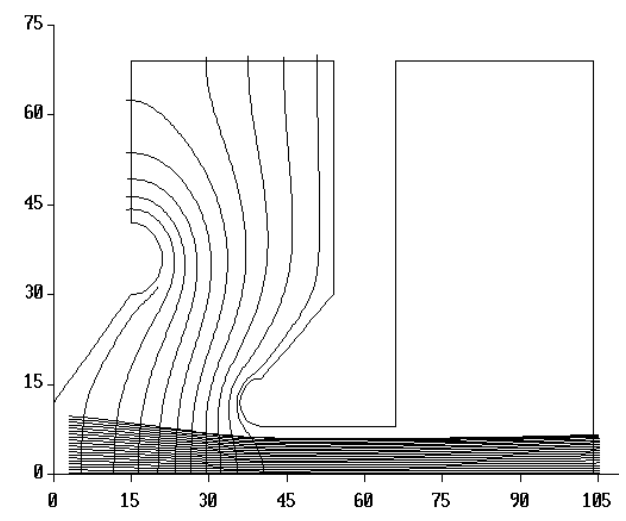

Figure 1: Electron beam trajectories in the GUN Voltage $=25 \mathrm{kV}, \mathrm{I}=1.02 \mathrm{~A}$.

\section{ACCELERATOR GUIDE}

A standing-wave constant-impedance type guide is selected for design. The guide length is selected as 561.5 mm long (3+9 cavities) to get $4.5 \mathrm{MeV}$ beam energy with available klystron power of 4.8 MW, for example TH-2154. The peak beam current for the optimized dose-rate from Eq.
(3) is $300 \mathrm{~mA}$. It can be easily obtained a Pierce-gun of $10.0 \mathrm{~mm}$ diameter $25 \mathrm{kV}$ and $1.02 \mathrm{~A}$. Buncher and acceleration cavities has been simulated using 2DSUPERFISH and 3D-HFSS codes. The SUPERFISH simulation shows the shunt impedance of $85 \mathrm{M} \Omega / \mathrm{m}$ for the acceleration cavity and $31 \mathrm{M} \Omega / \mathrm{m}$ for the buncher cavity. Two dimensional plane view of an accelerating cavity with detailed dimensions is shown in Fig. 2. The inner and outer diameters of the cavity are $84.0 \mathrm{~mm}$ and $100.0 \mathrm{~mm}$, respectively. The bore diameter is $20.0 \mathrm{~mm}$ while the length of coupling cavity and disk is $5.0 \mathrm{~mm}$ each. The period with an acceleration cavity, a coupling cavity, and two disks is $52.5 \mathrm{~mm}$. SUPERFISH simulation result of field contours of constant $\mathrm{H}_{\phi}$ is shown for acceleration and buncher cavities in Fig. 3. The cavity radius from SUPERFISH simulation for a particular resonance frequency has been then used in HFSS simulation to calculate eigenmodes.

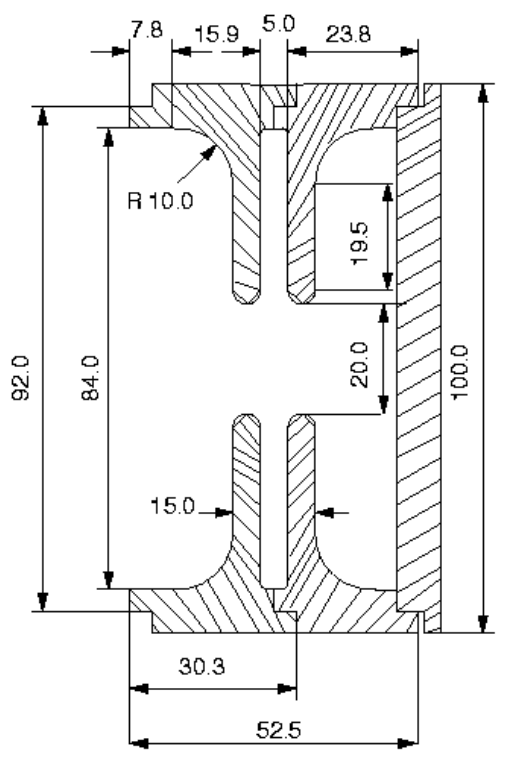

Figure 2: Acceleration cavity, unit in millimeter.
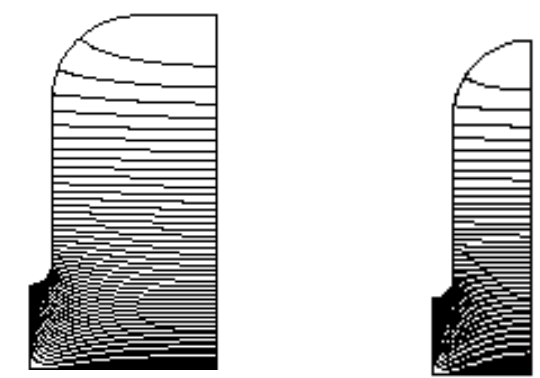

Figure 3: Field contours of constant $\mathrm{H}_{\phi}$ for accelerating (left) and buncher (right) cavities.

A schematic accelerating section is shown in Fig. 4. The lengths of E-GUN and buncher section are $129.5 \mathrm{~mm}$ and $105.0 \mathrm{~mm}$, respectively. The total length of the accelerator is $740.0 \mathrm{~mm}$. 


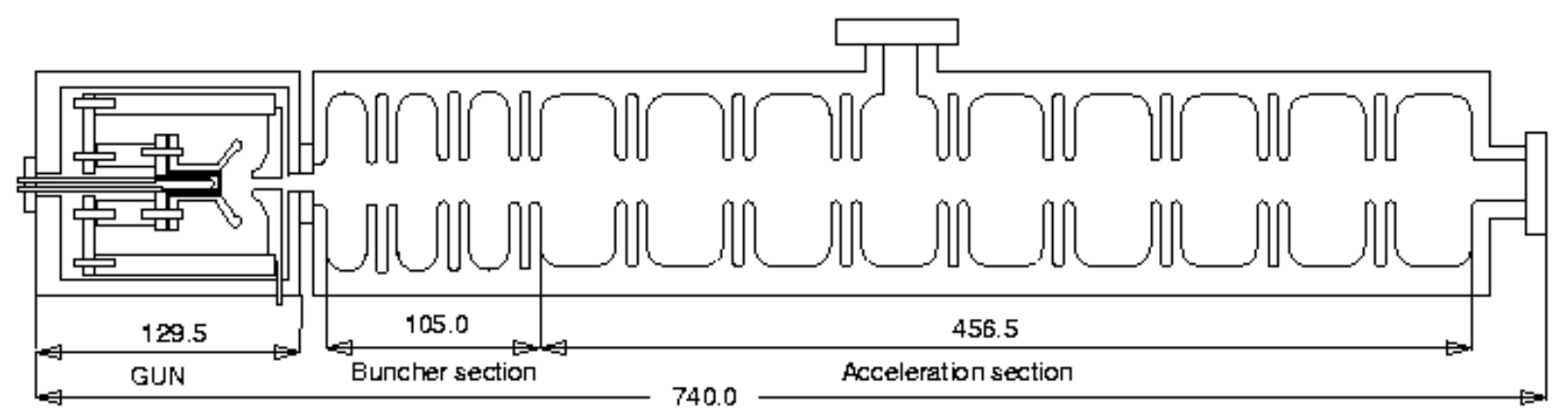

Figure 4: Schematic of SW linac, unit in millimeter.

\section{BEAM DYNAMICS}

The beam dynamics is evaluated with the computer code PAEMELA. Simulation results of phase space plots at the exit of the accelerator are shown in Fig. 5. It shows the longitudinal rms emittance of 37.3 degrees- $\mathrm{keV}$ at the exit of the accelerator with the beam diameter of $4.6 \mathrm{~mm}$.
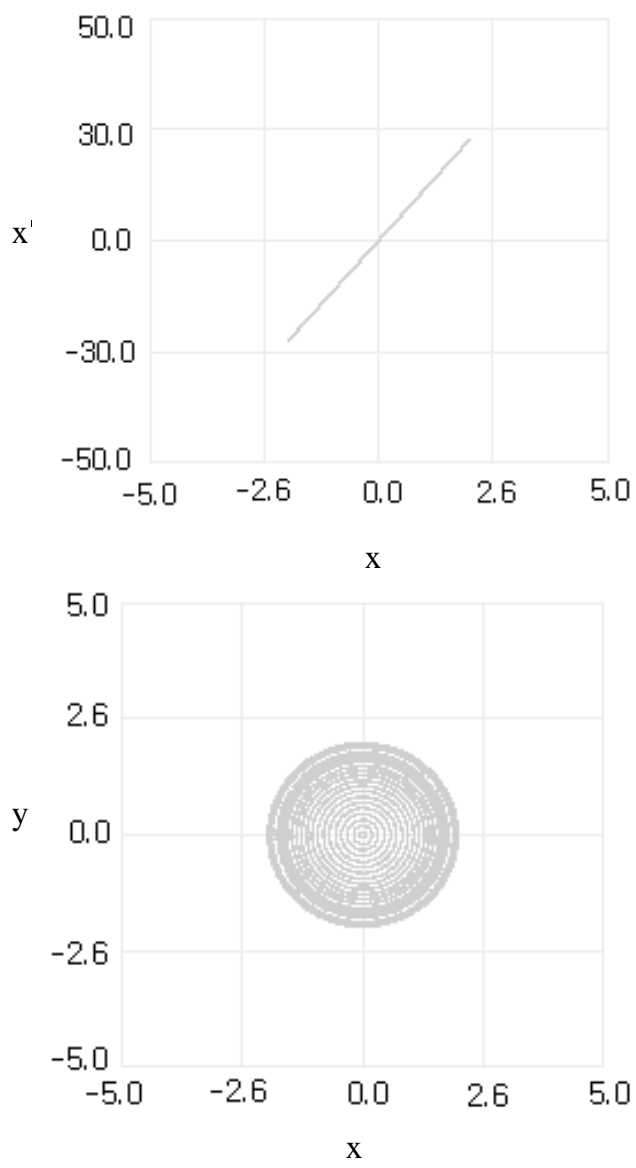

Figure 5: Phase space plot at the exit of the accelerator, units in millimeter.

\section{CONCLUSIONS}

The design study of a $4.5 \mathrm{MeV}$ standing-wave electron linac with peak power 1.4 MW has been carried out using computer codes SUPERFISH and PARMELA. The linac can give the average power of $6.5 \mathrm{~kW}$ with $5 \mu \mathrm{sec} / 960 \mathrm{pps}$ pulses. It can be used for polymer radiation processing, industrial irradiation, and recycling.

\section{ACKNOWLEDGEMENT}

Authors are grateful to Prof. T. Shintake of KEK for fruitful discussions and comments.

\section{REFERENCES}

[1] W. H. Scharf, in Biomedical Particle Accelerators (American Institute of Physics, New York, 1994), Chapter 1.

[2] S. O. Schriber, "Comparision of standing wave accelerators operating in the $2 \pi / 3$ and $\pi / 2$ modes," IEEE Trans. Nucl. Sci. 22, 1343 (1975).

[3] E. Tanabe, M. Bayer, and M. Trail, "A small diameter standing wave linear accelerator structure," IEEE Trans. Nucl. Sci. NS-32, 2975 (1985).

[4] T. Shintake, "Analysis of the transient response in periodic structures based on a coupled-resonator model" in Frontiers of Accelerator technology (Proc. of the Joint US-CERN-Japan International School, 918 September, 1996), edited by S. Kurokawa, M. Month, and S. Turner, p. 335.

[5] E. V. Kozyrev, "Standing-wave structures" in Frontiers of Accelerator technology (Proc. of the Joint US-CERN-Japan International School, 9-18 September, 1996), edited by S. Kurokawa, M. Month, and S. Turner, p. 339.

[6] H. B. Zhao, R. Carter, C. D. Hannaford, P. Zhou, and E. A. Hughes, "The improvement of the output performance of a low energy medical standing wave accelerator," Nucl. Instr. Meth. in Phys. Res. A 356, 552 (1995). 